\title{
Properties of Photocured Epoxy Resin Materials for Application in Piezoelectric Ultrasonic Transducer Matching Layers
}

\author{
Alexander Trogé ${ }^{1}$, Richard L. O'Leary ${ }^{2 *}$, Gordon Hayward ${ }^{2}$, Richard A. Pethrick ${ }^{1}$ and Anthony J. Mullholland ${ }^{3}$ \\ ${ }^{1}$ Department of Pure and Applied Chemistry, Thomas Graham Building, \\ University of Strathclyde, 295 Cathedral Street, Glasgow G1 1XL. \\ ${ }^{2}$ Centre for Ultrasonic Engineering, Dept. of Electronic and Electrical Engineering, Royal College Building, \\ University of Strathclyde, 204 George Street, Glasgow G1 1XW, Scotland \\ ${ }^{3}$ Department of Mathematics, Livingstone Tower, University of Strathclyde, 26 Richmond Street, \\ Glasgow G1 1XH
}

\begin{abstract}
This paper describes the acoustic properties of a range of epoxy resins prepared by photocuring that are suitable for application in piezoelectric ultrasonic transducer matching layers. Materials, based on blends of digylcidyl ether of Bisphenol A and 1,4-cyclohexanedimethanol diglycidyl ether, are described. Furthermore, in order to vary the elastic character of the base resin, samples containing polymer microspheres or barium sulphate particles are also described. The acoustic properties of the materials are determined by a liquid coupled through transmission methodology, capable of determining the velocity and attenuation of longitudinal and shear waves propagating in an isotropic layer. Measured acoustic properties are reported which demonstrate materials with specific acoustic impedance varying in the range 0.88 - 6.25 MRayls. In the samples comprising blends of resin types, a linear variation in the acoustic velocities and density was observed. In the barium sulphate filled samples, acoustic impedance showed an approximately linear variation with composition, reflecting the dominance of the density variation. While such variations can be predicted by simple mixing laws, relaxation and scattering effects influence the attenuation in both the blended and filled resins. These phenomena are discussed with reference to dynamic mechanical thermal analysis and differential scanning calorimetry of the samples.
\end{abstract}

\footnotetext{
* Author to whom correspondence should be addressed - Richard O'Leary, Centre for Ultrasonic Engineering, University of Strathclyde, 204 George Street, Glasgow, G1 1XW r.oleary@eee.strath.ac.uk
} 


\section{INTRODUCTION}

Piezoelectric transducers are used in a wide variety of ultrasonic applications in biomedicine, sonar and non-destructive evaluation. Typically, the properties of the transducer front end can be tailored to the specific task through appropriate design of acoustic matching layers and backing block to promote efficient and wideband operation. DeSilets et al [1] described a number of relationships for determining the required specific acoustic impedance of the matching layer(s) given the impedance of the transducer and load. For example, in the case of a transducer operating into a relatively low impedance load, such as water or biological tissue, it is commonplace to add a quarter wavelength matching layer whose impedance is determined from the geometric mean of the transducer and the load [1]. Where a wideband transducer is required, a single quarter wavelength matching layer should ideally possess a specific acoustic impedance, $Z_{m}$ :

$$
Z_{m}=\left(Z_{1} Z_{2}^{2}\right)^{1 / 3},
$$

Where $Z_{1}$ and $Z_{2}$ represent the impedance of the piezoelectric element and the load, respectively. More recently, Cannata et al [2] showed that for a system comprising two matching layers, the ideal inner, $Z_{m 1}$, and outer, $Z_{m 2}$, are found to be:

$$
\begin{aligned}
& Z_{m 1}=\left(Z_{1}^{4} Z_{2}^{3}\right)^{1 / 7}, \\
& Z_{m 2}=\left(Z_{1} Z_{2}^{6}\right)^{1 / 7} .
\end{aligned}
$$

Such relationships typically reflect some kind of theoretical optimality. However, in practice the availability of suitable materials with the desired specific acoustic impedance will govern the transducer design, resulting in some compromise being implemented, employing materials with the closest available match in terms of the specific acoustic impedance. In addition to matching layers applied to liquid, tissue or solid coupled probes, appropriately designed matching layers can be employed to facilitate gas coupling of piezoelectric devices. Various materials have been cited in the literature for use as air or gas coupled matching layers, such as silicone rubber $[3,4]$ or aero-gels of silica $[5,6]$, with varying degrees of success. 
In general, the motivation for acoustic matching is to enhance sensitivity or bandwidth of the piezoelectric transducer in a given application. Acoustic matching can be designed using discrete layers [1,2] or by employing a continuously varying impedance profile within the layer [7]. A recent study by the authors $[8,9]$ proposed a matching material that can be envisaged as a series of discrete layers wherein each layer possesses a slightly different acoustic impedance. Such a matching layer exhibits a profile of acoustic impedances through the thickness dimension, ranging from an acoustic impedance close to that of the transducer at the transducer side to an acoustic impedance close to the load at the load side of the layer. The impedance gradient of the matching layer is designed using a Chebyshev polynomial approach in order to reduce the degrees of freedom and arrive at an algorithm for a device design. Transducers designed using this approach can exhibit a threefold improvement, in terms of bandwidth, when compared to the standard single matching-layer design [9].

Polymer resins can be employed to manufacture layers with impedances in the range 2-3 MRayls $[2,10,11]$. In order to obtain materials exhibiting increased specific acoustic impedance, 0-3 connectivity composite materials, comprising a polymer resin and a particulate filler, can be used $[12,13,14]$. The properties of the $0-3$ composite obey a standard mixing rule $[12,13]$.

In this paper, a methodology is described to realise polymer materials with a range of elastic properties that are suitable for application in piezoelectric transducer and array assemblies. The approach allows the fabrication of polymer materials by successively photocuring thin layers of polymer $[15,16]$. The photocuring method can produce samples of homopolymers or a blend of homopolymers. Selection of a suitable particle filler also enables loaded systems to be produced. The materials investigated are based upon the glycidyl ether of bisphenol A (BisA) and the less well known 1,4-cyclohexanedimethanol diglycidyl ether (CHDG). BisA produces a rigid high glass transition $\left[\mathrm{T}_{\mathrm{g}}\right]$ material on cure whereas CHDG cures to a more flexible lower $\mathrm{T}_{\mathrm{g}}$ material. Combining these two basic resins systems allows the possibility of creating layers with acoustic properties designed to achieve optimum matching of the transducer to the media. The acoustic properties of BisA, cured with various amines, has been reported by a number of workers [17, $18,19,20,21,22]$. In the case of a photo cured epoxy resin system there is no additional aminecuring reagent, only a photo catalyst. Consequently, the physical properties of the materials in the current context will be very different from those of the amine cured materials previously investigated. Moreover, the method that is described in this paper cures the epoxy resin via 
opening of the epoxy rings to form an ether linked polymer backbone $[15,16]$. The structure of these polymers is therefore very different from those created by the conventional amine cure reaction, where the polymer backbone contains carbon nitrogen linkages. The use of blends of epoxy amine resins to vary the acoustic impedance of the passive phase in fabrication of piezoelectric composite transducers has been reported [23, 24]. Significant variations in the acoustic impedance were achieved by variation of the composition of the blend. The photopolymerisation method described here for the creation of epoxy resins has the advantage of allowing the rapid creation of micron thin films $[15,16]$. The samples presented have been formed in film format with thicker layers created by multiple progressive layer deposition.

In the following Section the materials and methodology employed to generate photo-cured epoxy resins are described. This is followed by a description of the method to carry out through transmission acoustic characterisation of the photo-cured materials. The results of the acoustic measurements are supported by dynamic mechanical thermal analysis (DMTA) and differential scanning calorimetry (DSC), in order to elucidate the behaviour of the materials as a function of temperature. Finally, in order to investigate the modification of the elastic character of the base resins, materials comprising low density gas filled microspheres or high density particulates encapsulated in a polymer resin are described. Incorporation of high density particles into the base resin allows, in principle, the acoustic impedance to be increased through an increase in the density and the propagation velocity. Likewise, the incorporation of hollow spheres creates the

possibility of lowering the acoustic impedance. However, dispersing particulate materials in a resin, can create scattering effects which can make prediction of the effects of changes in the acoustic properties difficult.

\section{Preparation OF PHOto-CURED POLYMERS}

The BisA material selected for this study has an epoxy equivalent of 182-192 g/eq and was obtained from Huntsman, Duxford, Cambridge, UK. CHDG has a lower molar mass but is more flexible than BisA and, as will be demonstrated in the course of this paper, has a lower glass transition temperature $\left(\mathrm{T}_{\mathrm{g}}\right)$. The photo-cure is initiated by a photoacid generator (PAG), in this case. The PAG and CHDG materials were obtained from Aldrich Chemical Co., Poole, Dorset, UK. The chemical structures of the BisA and CHGD monomers and the PAG material are shown 
in Figure 1. The structures of the monomers will lead to a variation in the resultant polymers. The BisA material, with the rigid aromatic ring structure, should result in higher values of acoustic velocity - when compared to the more flexible CHDG material. This will be discussed in more detail with reference to the measured data sets in the subsequent section of this paper. All of the materials were used in the form as supplied by the manufacturer.

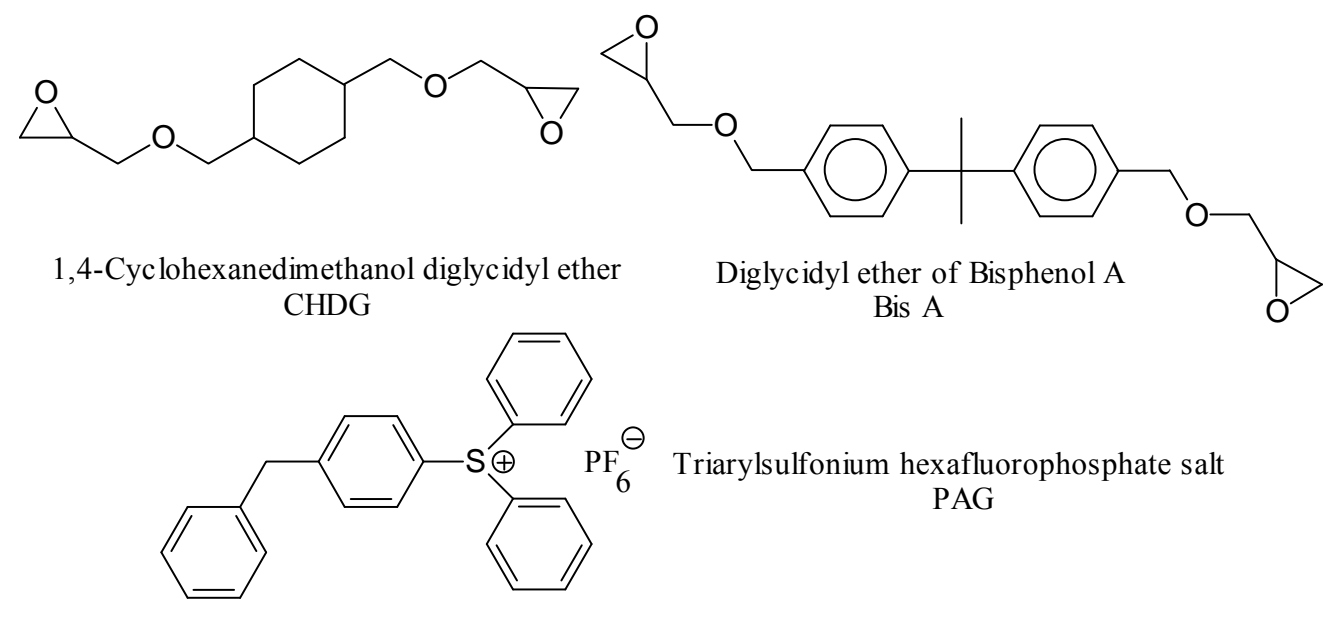

Figure 1 Chemical structures of the epoxy resins and the photo-initiator.

The solutions were prepared by thoroughly mixing $20 \mathrm{~g}$ of the monomer with $0.4 \mathrm{~g}$ of PAG and the mixture degassed under vacuum until no further bubbles were observed. Samples were prepared for study by placing $6.5 \mathrm{~g}$ of the mixture in a mould manufactured from polytetrafluoroethylene (PTFE), having interior dimensions of 50mm diameter and $6 \mathrm{~mm}$ depth. The mould was irradiated using a LIDAM Scientific ultraviolet (UV) lamp, at wavelength of $368 \mathrm{~nm}$ and $5 \mathrm{~mW} / \mathrm{cm}^{2}$ intensity. The thickness of the film was increased by subsequently curing $6.5 \mathrm{~g}$ aliquots of solution until the desired thickness was achieved. Samples of BisA and CHDG homopolymers were manufactured using this method, together with a range of samples containing a range of volume fractions of BisA in CHDG. It is important to note that the samples containing a volume fraction of BisA in excess of $49 \%$ were heated to $60^{\circ} \mathrm{C}$ before degassing to reduce the viscosity and allow any entrained air to be removed.

Samples of filled materials were manufactured using two different particles. To create low impedance materials Expancel PVDF hollow microspheres of $60 \mu \mathrm{m}$ diameter and a density of $60 \mathrm{~kg} / \mathrm{m}^{3}$ were used. Specimens were prepared using $10 \mathrm{~g}$ of CHDG with $0.2 \mathrm{~g}$ of PAG and 0.1, 
0.2, 0.3, 0.4 and $0.5 \mathrm{~g}$ of Expancel to achieve 16, 27, 36.5, 43.4 and $49 \%$ volume fraction of spheres, respectively. Higher impedance materials were produced using barium sulphate $\left(\mathrm{BaSO}_{4}\right)$, having a density of $4100 \mathrm{~kg} / \mathrm{m}^{3}$ and particle size in the range $8-12 \mu \mathrm{m}$. Specimens were produced containing 12, 18, 26, 35 and $51 \mathrm{~g}$ of $\mathrm{BaSO}_{4}$ with $20 \mathrm{~g}$ of BisA with $0.4 \mathrm{~g}$ of PAG to obtain 13, 19, 25, 31 and $40 \%$ volume fractions of $\mathrm{BaSO}_{4}$, respectively. The Expancel and $\mathrm{BaSO}_{4}$ materials were obtained from Boud Minerals (Sutton Bridge, Lincolnshire, UK).

\section{Acoustic Velocity MeAsurements}

Measurements were made at $500 \mathrm{kHz}$ by a through transmission method [19, 21, 25, 26, 27, 28]. All of the materials under test possess specific acoustic impedances close to water, making ultrasonic reflection/refraction measurements more difficult. Consequently the coupling fluid employed in the measurement technique is a low velocity oil. The material is a perfluorinated poly(ether) synthetic lubricant, sold under the trade name Krytox ${ }^{\circledR}$ (Du Pont Krytox ${ }^{\circledR}$ Synthetic

Lubricants, K. S. Paul Group, London, UK). The acoustic velocity, density and specific acoustic impedance of the liquid are $670 \mathrm{~ms}^{-1}, 1870 \mathrm{kgm}^{-3}$ and 1.25 MRayl, respectively. The velocity of the sample under consideration is determined by a narrowband through transmission time of flight technique. The theoretical consideration for the determination of the longitudinal and shear wave velocities will now be described.

\section{A. Determination of acoustic velocity}

The longitudinal velocity was determined using a pair transducers, each having a centre frequency of $500 \mathrm{kHz}$ and a $6 \mathrm{~dB}$ bandwidth of $200 \mathrm{kHz}$, with the sample configured such that the transmitted wavefront would be normally incident on the sample. The transducer and specimen were arranged to be axially aligned as shown in Figure 2, and detailed in the photograph of the apparatus. The sample holder is mounted on a high resolution rotary stage, supplied by Time and Precision Industries, (Hampshire, UK). The rotary stage gives an angular resolution of $0.01^{\circ}$ over $360^{\circ}$ and allows for the accurate alignment of the sample between the transmitting and receiving transducers. The transducers are used to acquire signals, transmitted through the coupling liquid, both without the sample and with the sample in place and normal to the transducers. Cross-correlation of the acquired signals allows the time difference for longitudinal propagation in the sample, $\Delta t_{l}$, to be calculated. Consider $D$ is the transducer 
separation, $c_{i}$ is the velocity in the coupling medium, $d$ is the sample thickness, $c_{l}$ is the longitudinal velocity in the sample, we may write an expression for $\Delta t_{l}$

$$
\Delta t_{l}=t_{1}-t_{2}=\frac{D}{c_{i}}-\left(\frac{D-d}{c_{i}}+\frac{d}{c_{l}}\right)=\frac{d}{c_{i}}-\frac{d}{c_{l}}
$$

Determination of the shear wave velocity is carried out in a similar fashion; the sample is rotated until only the shear wave is propagated in the sample. This condition will not occur if $c_{i}$ is greater than $c_{l}$, in the case of polymer material this is often the case and is the reason for employing the low velocity coupling medium Krytox rather than water in the experimental arrangement. As before acquiring signals transmitted through the coupling liquid, both without the sample and with the sample in place under conditions of shear wave propagation cross correlation can be employed to determine the time difference for shear wave propagation in the sample, $\Delta t_{s}$. An expression for $\Delta t_{s}$ can be written as follows [28]

$$
\Delta t_{s}=d\left(\frac{\cos \theta_{i} \sqrt{1-\frac{c_{s}{ }^{2} \sin ^{2} \theta_{i}}{c_{i}^{2}}+\frac{c_{s} \sin ^{2} \theta_{i}}{c_{i}}}}{c_{i} \sqrt{1-\frac{c_{s}{ }^{2} \sin ^{2} \theta_{i}}{c_{i}^{2}}}}-\frac{1}{c_{s} \sqrt{1-\frac{c_{s}{ }^{2} \sin ^{2} \theta_{i}}{c_{i}^{2}}}}\right) .
$$

Where $c_{s}$ is the shear wave velocity in the sample and $\theta_{i}$ is the incident angle at which the signal was acquired, determined from the rotary stage, $c_{s}$ in the sample can be found using a numerical solution of Equation 5. Since the materials under study are isotropic, the complete compliance and stiffness matrices and the salient moduli of the material be calculated from $c_{l}$ and $c_{s}$ and the sample density, $\rho$,. [31, 32].

\section{B. Experimental Measurement of Acoustic Properties}

For convenience, the experimental methodology for the determination of the longitudinal and shear wave velocities and attenuation will be described using a sample of photocured CHDG. The data obtained for the other samples will then be presented in tabular form. 


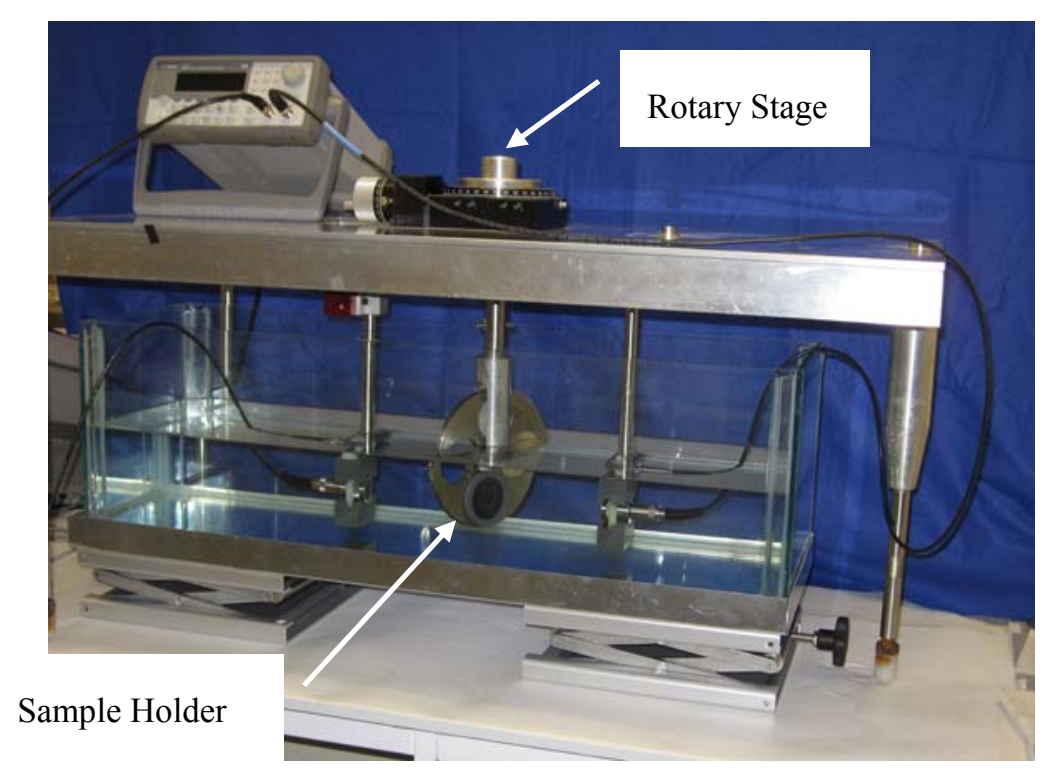

Figure 2 Photograph of the experimental apparatus

The transducers employed in the measurements were a pair of matched devices, operating at $500 \mathrm{kHz}$ with a $6 \mathrm{~dB}$ bandwidth of $200 \mathrm{kHz}$, supplied by Alba Ultrasound Ltd, Glasgow, Scotland. The sample is positioned in the far-field of both devices, in order to approximate plane wave conditions.

The CHDG sample was placed in the sample holder - this was carried out on a surface table to ensure that the sample was correctly aligned in the holder. The sample holder was then mounted in the apparatus, with the sample axially aligned with the transducers. The angle was then adjusted to ensure the sample faces were parallel with the transducer faces under conditions of normal incidence. The velocity of the coupling medium and the signal transmitted through the coupling medium alone were recorded using the digitising oscilloscope. In the remainder of the analysis, this signal will be termed the reference signal. With the sample under consideration between the transducers, the signal transmitted through the specimen was recorded at normal incidence - this signal is the longitudinal transmitted signal. The reference signal and the longitudinal transmitted signal are then correlated in the time domain using Matlab, in order to determine the time difference, $\Delta t_{l}$ in Equation 4. Since the other sample thickness and the incident velocity can be measured the longitudinal velocity in the sample, $c_{l}$, can be determined by rearranging Equation 4. The sample is then rotated until, and provided the $c_{l}$ is greater than $c_{i}$ a shear wave is propagated into the sample. The angle of incidence is adjusted until the signal 
amplitude reaches a maximum, the received signal and the angle of incidence ( $30.4^{\circ}$ in this case) are both recorded. As before, the time domain signals are cross-correlated using Matlab to determine the time difference between the two signals in order to determine, $\Delta t_{s}$ in Equation 5. The time data can then be used to calculate the longitudinal and shear wave velocities. In the case of the CHDG sample the longitudinal and shear wave velocities were found to be $1784.8 \mathrm{~m} / \mathrm{s}$ and $766.3 \mathrm{~m} / \mathrm{s}$, respectively.

\section{Determination of Longitudinal and Shear Wave Attenuation}

Since the velocities in the material have been determined, the appropriate velocity data can then be used in order to determine the attenuation of the individual wave types by employing a methodology similar to that reported by Selfridge [29], extended to consider oblique incidence [28] and so include the contribution of mode conversion at the fluid-solid boundary at the front-face of the sample and solid-fluid interface at the rear of the sample.

Following Brekhovskikh [30], the transmission coefficient for a fluid coupled isotropic solid layer, for lossless longitudinal waves can be written

$$
D_{l}=W \frac{c_{i} \cos \theta_{l}}{c_{l} \cos \theta_{i} \cos ^{2} 2 \theta_{s}}\left(1-\frac{Z+Z_{s} \sin ^{2} 2 \theta_{s}-Z_{l} \cos ^{2} 2 \theta_{s}}{Z+Z_{s} \sin ^{2} 2 \theta_{s}+Z_{l} \cos ^{2} 2 \theta_{s}}\right) .
$$

Where

$$
\begin{aligned}
& W=\frac{\rho}{\rho_{1}} \frac{2 Z_{l} \cos 2 \theta_{s}}{Z_{l} \cos ^{2} 2 \theta_{s}+Z_{s} \sin ^{2} 2 \theta_{s}+Z} \\
& Z=\frac{\rho c_{i}}{\cos \theta_{i}}, Z_{l}=\frac{\rho_{1} c_{l}}{\cos \theta_{l}} \text { and } Z_{s}=\frac{\rho_{1} c_{s}}{\cos \theta_{s}}
\end{aligned}
$$

and the densities of the fluid and sample are $\rho$ and $\rho_{l}$, respectively. Similarly the transmission coefficient for a fluid coupled isotropic solid layer, for lossless shear waves, $D_{s}$, can be written 


$$
D_{s}=P \frac{\tan \theta_{i}}{2 \sin ^{2} \theta_{s}}\left(1-\frac{Z+Z_{l} \cos ^{2} 2 \theta_{s}-Z_{s} \sin ^{2} 2 \theta_{s}}{Z+Z_{l} \cos ^{2} 2 \theta_{s}+Z_{s} \sin ^{2} 2 \theta_{s}}\right) .
$$

Where

$$
P=-\frac{\rho}{\rho_{1}} \frac{2 Z_{s} \sin 2 \theta_{s}}{Z_{l} \cos ^{2} 2 \theta_{s}+Z_{s} \sin ^{2} 2 \theta_{s}+Z}
$$

And the other symbols are as previously defined. The losses associated with the reflection and transmission at the boundaries in a fluid coupled isotropic layer for longitudinal and shear waves are defined by Equations 6 and 8, respectively. Modifying Equations 6 and 8 to incorporate an exponential loss term will therefore accommodate the attenuation of each wave type as it is propagated through the sample $[28,31]$

$$
\begin{gathered}
D_{l}=W e^{\left(\frac{-\alpha_{l} d}{\cos \theta_{l}}\right)} \frac{c_{i} \cos \theta_{l}}{c_{l} \cos \theta_{i} \cos ^{2} 2 \theta_{s}}\left(1-\frac{Z+Z_{s} \sin ^{2} 2 \theta_{s}-Z_{l} \cos ^{2} 2 \theta_{s}}{Z+Z_{s} \sin ^{2} 2 \theta_{s}+Z_{l} \cos ^{2} 2 \theta_{s}}\right), \\
D_{s}=P e^{\left(\frac{-\alpha_{s} d}{\cos \theta_{s}}\right)} \frac{\tan \theta_{i}}{2 \sin ^{2} \theta_{s}}\left(1-\frac{Z+Z_{l} \cos ^{2} 2 \theta_{s}-Z_{s} \sin ^{2} 2 \theta_{s}}{Z+Z_{l} \cos ^{2} 2 \theta_{s}+Z_{s} \sin ^{2} 2 \theta_{s}}\right),
\end{gathered}
$$

where $\alpha_{l}$ and $\alpha_{s}$ are the attenuation factors in nepers per metre of the longitudinal and shear waves, respectively. These equations can then be used to determine attenuation of the longitudinal and shear waves, since the appropriate acoustic velocities in the sample can be determined using the method presented previously. The experimentally measured transmitted amplitude data for both the longitudinal and shear waves can then be compared to the theoretically determined transmission coefficient of the associated wave type using Equations 10 and 11. The loss term in the appropriate equation can then be adjusted order to determine the attenuation of the wave type propagating in the sample as a function of incident angle. 
In order to quantify attenuation in the sample, the velocity of the coupling medium was measured, as was the peak-to-peak voltage of the signal transmitted through the coupling medium. The sample was then aligned in the holder as before and the peak-to-peak voltage of the signal transmitted through the sample measured using the oscilloscope, from normal incidence over a range of incident angles large enough to encompass both longitudinal and shear wave propagation in the sample, with measurements taken at one-degree intervals. The experimental data obtained undergoes numerical manipulation to account for the amplitude of the reference signal, such that the amplitude incident at the sample can be considered to be unity.

The theoretical transmission coefficient was calculated from the appropriate velocities and densities over the angular range of interest using Equations 10 and 11. The theoretical and experimental data is plotted together for comparison. The figure for attenuation of each wave type, $\alpha_{l}$ and $\alpha_{s}$ in Equations 10 and 11, respectively, is then adjusted and the theoretical transmission coefficient recalculated and compared to the experimentally measured data. This process is repeated until the best fit is obtained between the theoretical and experimental data. Figure 3 details a plot of the theoretically determined transmission coefficients for the longitudinal and shear wave considering attenuation in the sample of CHDG; the experimentally measured amplitude data is also plotted for comparison. The error bars in experimental data show in Figure 3 are determined from the accuracy of measuring the peak-to-peak voltage using the oscilloscope. The curve fitting approach, covering the complete data set, produces a more robust result since the entire range of incident angles is being considered. In the case of the CHDG material the longitudinal and shear wave attenuation were found to be $573 \mathrm{~dB} / \mathrm{m}$ and $6228 \mathrm{~dB} / \mathrm{m}$ at $500 \mathrm{kHz}$. The measurements were undertaken at this frequency, since the materials were intended for use in device designs with a nominal centre frequency of $500 \mathrm{kHz}$. The methodology described, is applicable across a wide range of frequencies, only the pair of transducers need to be substituted in order to perform measurements at different frequencies. It is important to note, that the attenuation in the materials under investigation would increase with increasing frequency. In addition, velocity dispersion will also be observed as the centre frequency of the measurement is altered, although to a significantly lesser extent than in the case of loss [32]. 

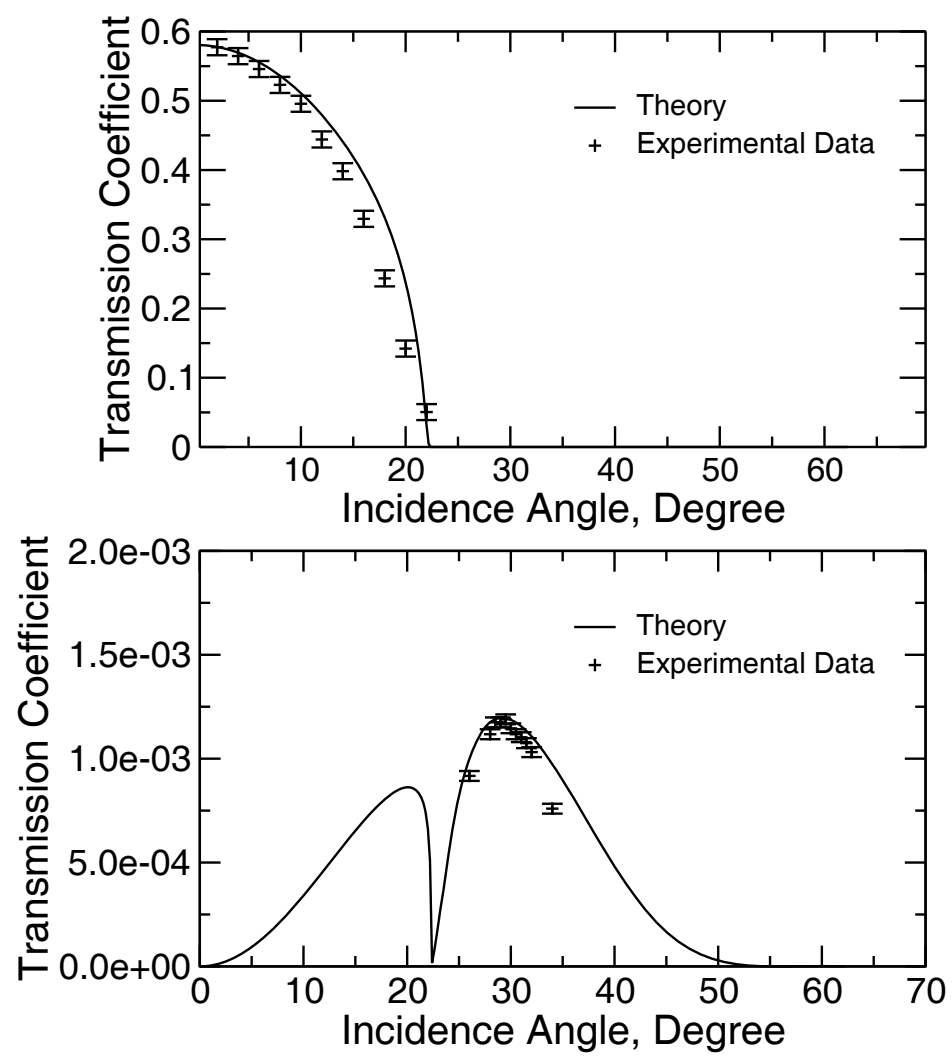

Figure 3 Curve Fitting for the determination of attenuation in CHDG, upper plot - Longitudinal data and lower plot - Shear data

\section{Acoustic and Mechanical Properties of Polymer Blends}

In this paper we are interested in measuring the variation of physical properties for CHDG Bis A blends. The data presented in Table 1 are for a range of formulations - the $0 \%$ volume fraction BisA in CHDG represents the CHDG homopolymer. The density data detailed in Table 1 was determined using a flotation method [21]. The density of the mixture for which the polymer sample just floats was determined using an Anton Paar Digital DMA601 density-measuring cell and a DMA60 density meter (Stanton Redcroft, London). A saturated solution of potassium bromide was used for measurements on the high-density materials and a mixture of nhexane/dichloromethane was used for low-density materials. The experimentally determined data detailed in Table 1 was subject to uncertainty analysis determined by propagation of errors [33] for Equations 4 and 5 and the associated calculations for acoustic impedance and elastic moduli [31] in order to determine the error bars that will be employed in the ensuing Figures. 


\begin{tabular}{|c|c|c|c|c|c|c|c|c|c|c|c|}
\hline $\begin{array}{c}\text { Formulation } \\
\text { Volume Fraction of } \\
\text { BisA in CHDG }\end{array}$ & $\mathbf{0}$ & 9.8 & 19.7 & 29.6 & 40.0 & 50.0 & 59.6 & 69.6 & 79.7 & 89.8 & 100 \\
\hline$c_{l}\left(m \cdot s^{-1}\right)$ & 1784.8 & 1956.3 & 2003.2 & 2091.0 & 2232.6 & 2246.9 & 2356.8 & 2392.7 & 2441.4 & 2531.0 & 2568.3 \\
\hline$c_{s}\left(m \cdot s^{-1}\right)$ & 766.3 & 800.4 & 799.5 & 870.7 & 910.5 & 924.0 & 943.8 & 971.9 & 1004.7 & 1048.5 & 1092.7 \\
\hline Density $\left(\mathrm{kg} \cdot \mathrm{m}^{-3}\right)$ & 1157.3 & 1156.0 & 1158.8 & 1152.9 & 1162.0 & 1172.4 & 1178.0 & 1181.0 & 1170.8 & 1180.9 & 1177.9 \\
\hline Poisson's Ratio & 0.387 & 0.400 & 0.405 & 0.395 & 0.400 & 0.398 & 0.405 & 0.401 & 0.398 & 0.396 & 0.390 \\
\hline Z (MRayls) & 2.066 & 2.261 & 2.321 & 2.411 & 2.594 & 2.634 & 2.776 & 2.826 & 2.858 & 2.989 & 3.025 \\
\hline E (GPa) & 1.885 & 2.073 & 2.082 & 2.439 & 2.698 & 2.799 & 2.948 & 3.126 & 3.305 & 3.626 & 3.908 \\
\hline K (GPa) & 2.780 & 3.437 & 3.662 & 3.875 & 4.508 & 4.584 & 5.144 & 5.274 & 5.403 & 5.834 & 5.894 \\
\hline G (GPa) & 0.679 & 0.741 & 0.741 & 0.8740 & 0.963 & 1.001 & 1.049 & 1.116 & 1.182 & 1.298 & 1.406 \\
\hline$\alpha_{1}\left(d B \cdot m^{-1}\right)$ & 573 & 426 & 252 & 174 & 156 & 130 & 52 & 43 & 26 & 35 & 35 \\
\hline$\alpha_{s}\left(d B \cdot m^{-1}\right)$ & 6228 & 5993 & 4334 & 3839 & 2562 & 2493 & 1729 & 1416 & 973 & 643 & 356 \\
\hline $\operatorname{Tg}\left({ }^{\circ} \mathrm{C}\right)$ & $23 \pm 1$ & $30 \pm 1$ & $35 \pm 1$ & $44 \pm 1$ & $48 \pm 1$ & $52 \pm 1$ & $57 \pm 1$ & $61 \pm 1$ & $65 \pm 1$ & $66 \pm 1$ & $62 \pm 1$ \\
\hline
\end{tabular}

Table 1 Mechanical and acoustic properties of the BisA-CHDG mixture polymers determined by through transmission where $c_{l}$ is the longitudinal velocity, $c_{s}$ the shear velocity, $Z$ the acoustic impedance, $E$ the Young modulus, $K$ the bulk modulus, $G$ the shear modulus, $\alpha_{l}$ the longitudinal attenuation and $\alpha_{s}$ the shear attenuation. 
As would be expected, the elastic moduli of the materials detailed in Table 1vary linearly with the material composition and can be described by an equation of the form:-

$$
E_{e f f}=\phi \times E_{1}+(1-\phi) E_{2}
$$

where $E_{\text {eff }}$ is the Young's modulus of a composition of two polymers, $P_{1}$ and $P_{2}$, Young's moduli in the pure state are represented by $E_{1}$ and $E_{2}$, respectively, and $\phi$ is the volume fraction of $P_{1}$ in the composition. Expressions, taking the same form as Equation 12, can be written for both the shear and bulk moduli. Figure 4 illustrates the experimentally determined values for the Young's, shear and bulk moduli of the materials described in Table 1 and compares each data set to the respective mixing rule. In each case effective modulus is calculated using the mixing rule and the appropriate modulus of the BisA and CHDG materials in their pure state. The BisA material generates a more highly packed morphology, as a result of the rigid aromatic ring structure in the monomer, shown in Figure 1. This generates a closely packed chain morphology, resulting in higher modulus values for the BisA than the more flexible CHDG. It is clear from Figure 4, that the mixing rule defined in Equation 12 describes the behaviour of either the longitudinal or shear wave velocity as the composition is varied. 


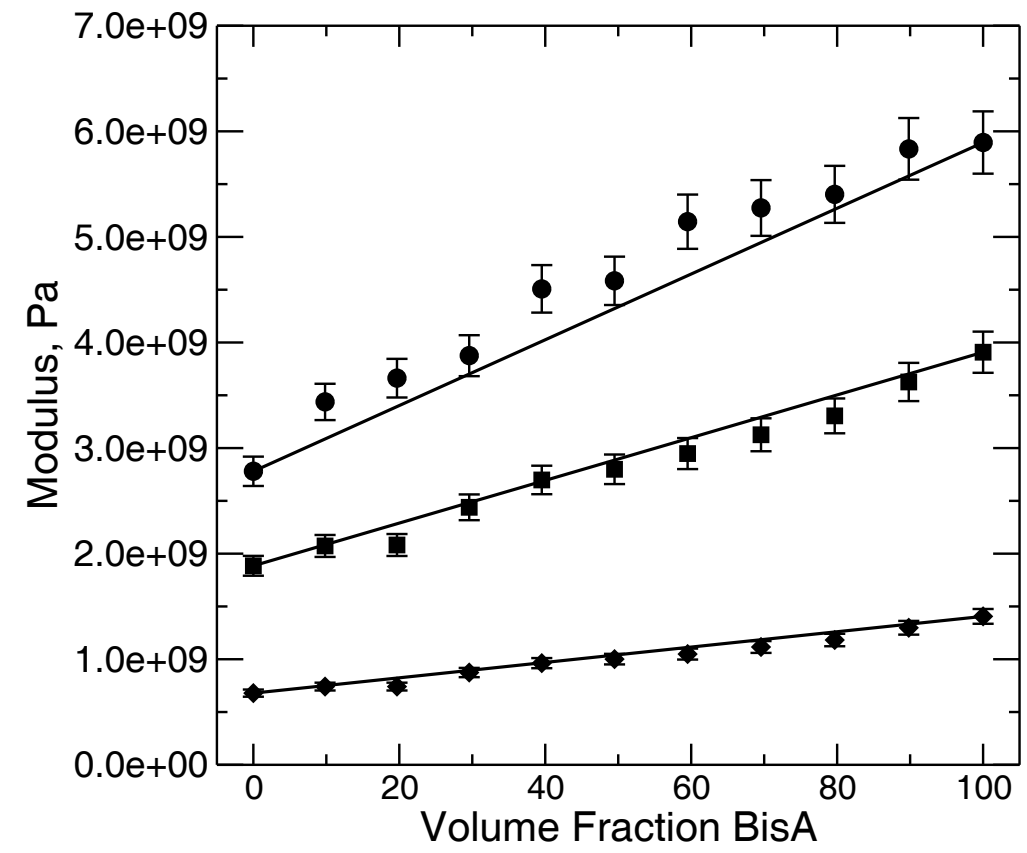

Figure 4 Comparison of experimentally determined moduli as the composition is varied,

- Bulk modulus, $\boldsymbol{\Delta}$ Young's modulus, Shear modulus and - data derived from the mixing rule.

Error bars determined by propagation of uncertainty

In a similar fashion, an expression for the variation in density as a function of the composition can be written as follows,

$$
\rho_{\text {eff }}=\phi \rho_{1}+(1-\phi) \rho_{2}
$$

Where $\rho_{\text {eff }}$ is the density of the composition; $\rho_{1}$ and $\rho_{2}$ are the densities of the component polymers $P_{1}$ and $P_{2}$ in the pure state, respectively. As before $\phi$ is the volume fraction of $P_{1}$ in the composition. Since the intended application of the materials is in the construction of acoustic matching layers it is of interest to derive and expression for the variation in velocity and hence acoustic impedance. The bulk and shear moduli of an isotropic material can be expressed in terms acoustic velocities as follows [23]

$$
K=\rho\left[c_{l}^{2}-\frac{4}{3} c_{s}^{2}\right] . \text { and } G=\rho c_{s}^{2}
$$


Where $K$ is the bulk modulus and $G$ is the shear modulus and the other symbols are as previously defined. An expression for longitudinal velocity can be written as follows

$$
c_{l}=\sqrt{\frac{\left(K+\frac{4}{3} G\right)}{\rho}} .
$$

Employing Equations 12, 13 and 15 and the appropriate moduli and density data for BisA and CHDG in the pure state, the variation in acoustic impedance as a function of composition can be determined - the experimentally determined data and that calculated using the mix

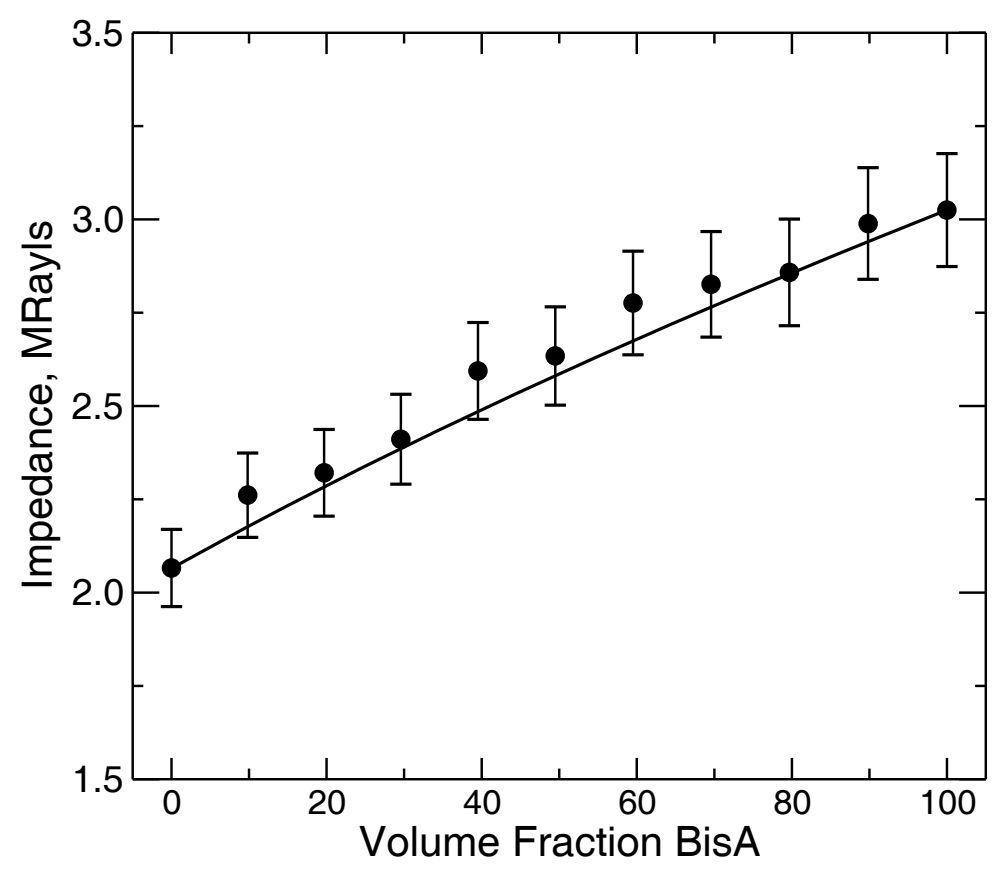

Figure 5 Variation in acoustic impedance of the polymer blend as the composition is varied - Measured Acoustic Impedance and - Acoustic Impedance data derived from the mixing rule. Error bars determined by propagation of uncertainty

Considering the acoustic impedance data, detailed in Table 1 and plotted in Figure 5, indicates that it is possible to achieve impedances in the range 2 to 3 MRayls by choosing appropriate proportions of monomers in the mixture. The longitudinal and shear attenuation however, shows a more complex variation as a function of composition. 
Figure 6 details a plot of the $\log$ of the attenuation versus composition for both longitudinal and shear wave attenuation. The slope coefficient for the longitudinal attenuation is -0.03783 and -0.02332 for shear attenuation. The plots shown in are linear over the range $0-80 \%$ volume fraction BisA for both the longitudinal and shear attenuation.
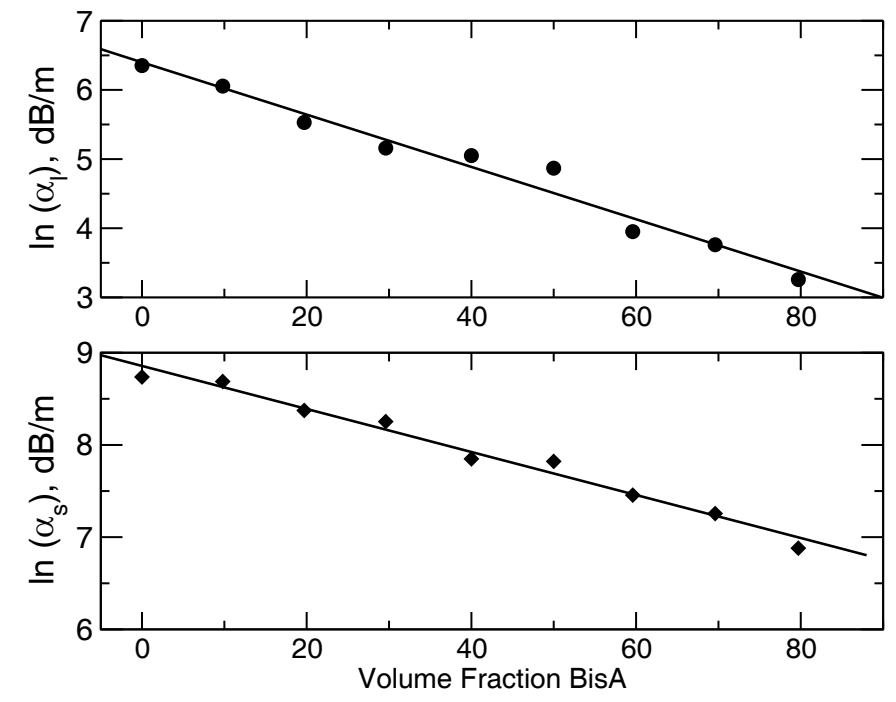

Figure 6 Variation in attenuation measured at $500 \mathrm{kHz}$ as a function of composition,

- Longitudinal Attenuation, Shear Attenuation and - linear fit determined by least squares method

\section{Thermal Analysis of Polymer Blends}

To further investigate the materials dynamic mechanical thermal analysis (DMTA) and differential scanning calorimetry (DSC) was also employed. The results of these analyses for the BisA - CHDG blends will now be described. A Polymer Laboratories DMTA (Church Stretton, Cheshire, UK) was used to measure the modulus and loss over the temperature range from -140 to $150{ }^{\circ} \mathrm{C}$ at an oscillatory frequency of $1 \mathrm{~Hz}$ using a single cantilever mode. Measurements were performed using strips $4 \mathrm{~mm}$ wide, 1.5 to $2 \mathrm{~mm}$ thick and $25 \mathrm{~mm}$ in length. A clamp torque from 10 to $40 \mathrm{~N}$ was used, depending on the hardness of the sample at room temperature. The method used has been described previously [21,34]. DMTA analysis of samples with $0-100 \%$ volume fraction of BisA are shown in Figure 7, where the 0\% volume fraction BisA represents 100\% CHGD. The low temperature modulus decreases with increasing CHDG content, apart for the initial 
$20 \%$ volume fraction BisA that exhibits a higher value. The $\mathrm{Tg}$ is also increased for this sample and then decreases progressively with increasing CHDG content. Similar behaviour was observed for variation of the rubbery modulus. The $\tan \delta$ plots indicate that the glass transition becomes broader as the amount of CHDG is increased. The boatchair isomerisation of polymers containing cyclohexane groups has been studied previously using DMTA for various polymers containing cyclohexane groups [35]. The location of the $\tan \delta$ peaks for the CHDG ring, Figure 7(b), occurs at a significantly higher temperature than that for polycyclohexylmethacrylate, wherein the $\tan \delta$ peaks occurs at $-100^{\circ} \mathrm{C}$ [35]. In the CHDG material the peak in tan $\delta$, occurring at approximately $0{ }^{\circ} \mathrm{C}$, reflects the restrictions imposed by the cyclohexane ring being linked at both ends into the matrix. Incorporation of the cyclohexane ring of the CHDG in the BisA matrix produces plasticization, as a result there is a progressive decrease in the temperature at which the peak in $\tan \delta$ occurs with increasing CHDG content.

DSC measurements were performed using a TA Q1000 DSC instrument (TA Instruments, Crawley, UK). A sample of polymer, nominal mass $5 \mathrm{mg}$, was placed in a sealed aluminium pan and heated from -50 to $150{ }^{\circ} \mathrm{C}$ at a rate of $10{ }^{\circ} \mathrm{C}$ per minute under a flow of $20 \mathrm{ml} \cdot \mathrm{min}^{-1}$ of nitrogen gas. As before, the method used has been described previously $[16,34]$. The results of the DSC analysis for the $0-100 \%$ volume fraction of BisA are shown in Figure 8. The data confirm the decrease in the $\mathrm{Tg}$ with increasing CHGD content. A stress peak is observed for the 60,80 and $100 \%$ volume fraction BisA just after the glass transition temperature reflecting the effects of shrinkage of the sample during the polymerisation process. The more flexible CHDG does not exhibit this peak indicating that stress relaxation can occur more readily as a result of increased flexibility. 

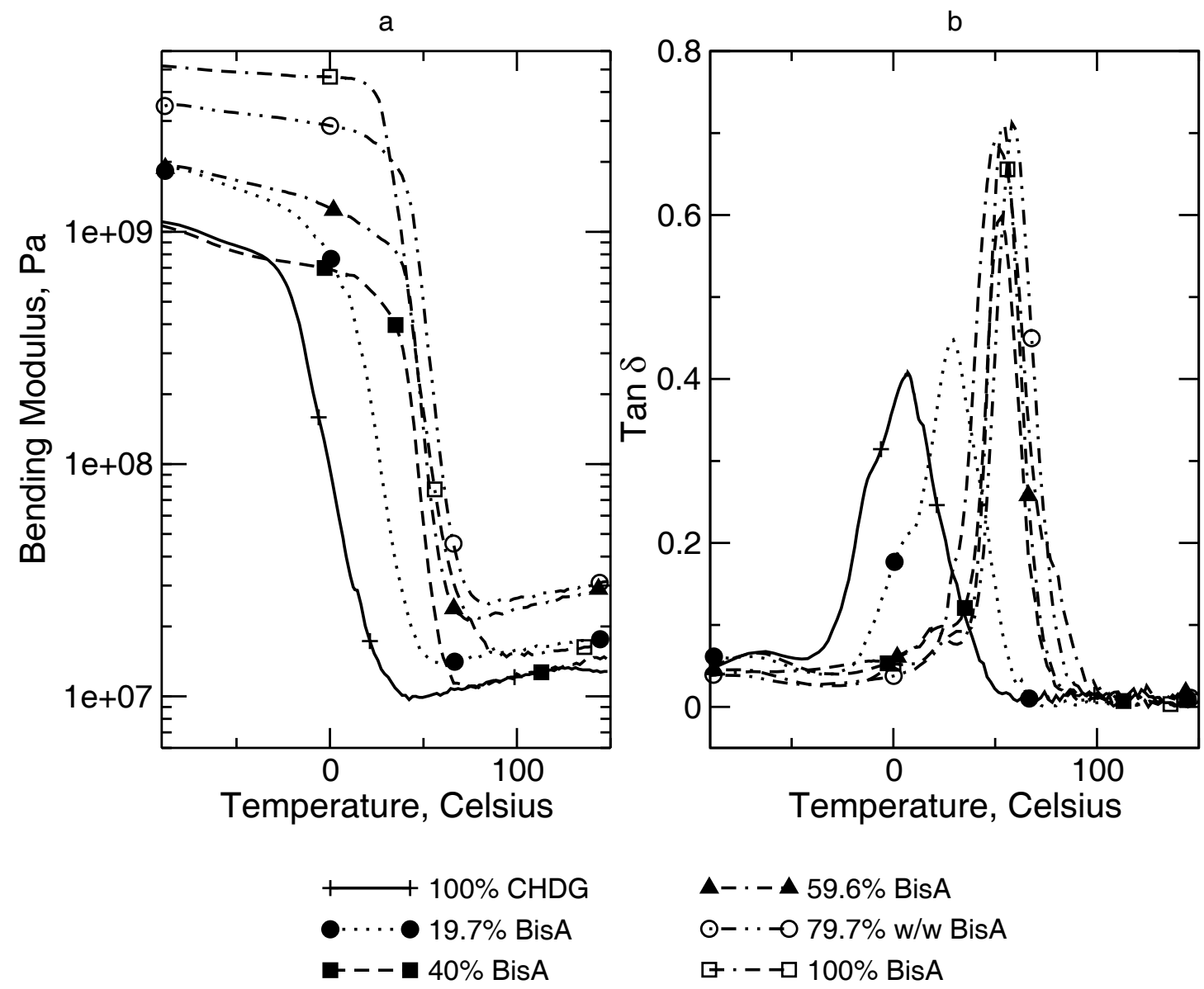

Figure 7 DMTA traces of the BisA-CHDG mixtures measured at $1 \mathrm{~Hz}$;

(a) bending modulus and (b) Tan $\delta$. 


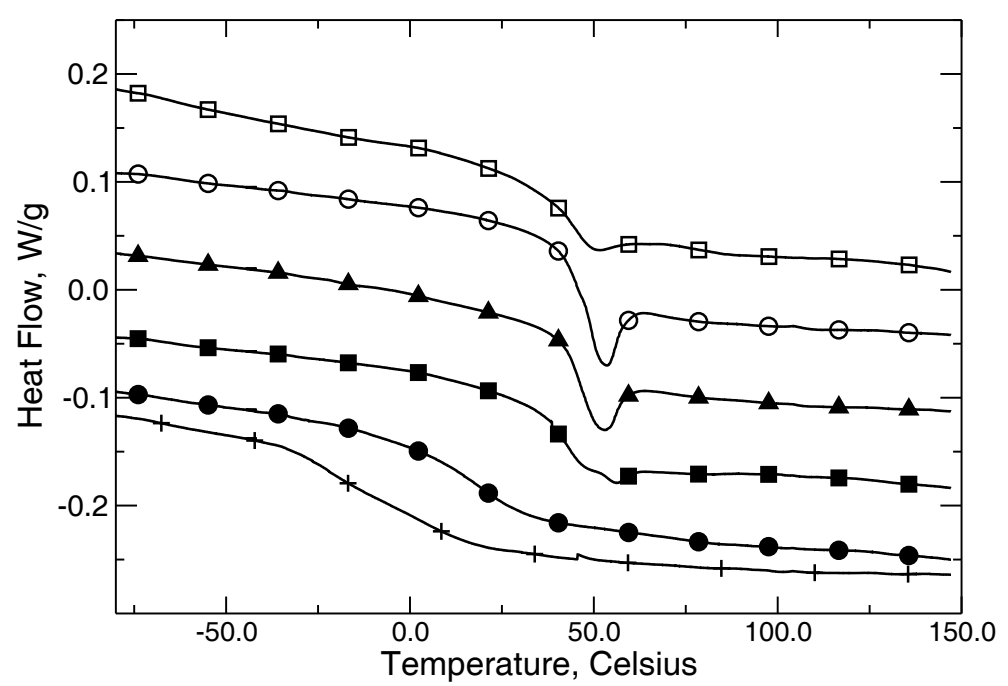

Figure 8 DSC traces of the BisA-CHDG mixture measured at a temperature ramp of $10^{\circ} \mathrm{C} / \mathrm{min}$.

+ 100\% CHDG, - 19.7\% BisA, - 40\% BisA, \ 59.6\% BisA, O 79.7\% BisA, 口 100\% BisA.

The variation observed in the attenuation as a function of composition is in line with the variation in the $\mathrm{T}_{\mathrm{g}}$ and reflects the contribution from the facile boat - chair ring inversion for the CHDG monomer. Increasing BisA content appears to suppress the boat-chair relaxation, evidenced by the increasingly smaller changes in the $T_{g}$ and the attenuation data. The BisA does not have a comparable relaxation process and therefore does not contribute significantly to the attenuation.

To summarise, for acoustic data measured at $500 \mathrm{kHz}$, the salient elastic moduli and attenuation coefficients, for a range of binary photocured epoxy systems have been presented. The velocity and elastic moduli are shown to follow a general linear trend as a function of polymer composition. Analysis of the attenuation exhibited by the range of materials is found to be in line with the $T_{g}$ data. A range of materials with specific acoustic impedance in the range 2-3 MRayls have been described. These materials are of use in the construction of piezoelectric probes. In order to further explore the range of elastic properties that can be achieved, 0-3 connectivity composite materials based on the photocured systems already described were prepared. The type of particulate addition to the resin will determine whether the 0-3 connectivity material undergoes an increase or decrease in the specific acoustic impedance, from that of the pure resin. The addition of 
air filled microspheres and inorganic salts of barium have been investigated and the behaviour of these materials will now be dealt with in turn.

\section{LOW DENSITY FILLED SYSTEMS}

To investigate the potential of the photocured resin systems to produce materials exhibiting low acoustic impedance, a number of formulations incorporating low density Expancel microspheres, obtained from Boud Minerals (Sutton Bridge, Lincolnshire, UK), were investigated. Expancel microspheres comprise polyvinylidene fluoride (PVDF) shell filled with a gas. A range of samples were prepared using the CHDG material as the host matrix and the percentage of Expancel spheres incorporated in the resin varied in the range $16-49 \%$ volume fraction. For low filler loadings, when the polymerization was carried out with the pure monomer, phase separation was observed in the resulting cured polymer. To avoid this problem, the resin was pre-polymerized to increase the viscosity so that phase separation did not occur. This phenomenon will be discussed in more detail in relation to the DMTA of these materials. It is also important to note that it was not possible to create viable films with greater than $49 \%$ volume fraction of Expancel. At such loadings the polymerisation was inhibited by the large volume of Expancel in the formulation. As before, the mechanical properties of the materials were measured using the through transmission method. Table 2 summarises the results. The incorporation of the microspheres leads to the predicted reduction in impedance. The longitudinal attenuation in these materials is very high, and increases with increasing microsphere content - the attenuation reflects multiple scattering of the sound wave propagating in the samples. Similar phenomena have been observed for polymers loaded with glass spheres [36] and talc filler [37]. In the materials presented the wavelength is in the range 2.9 - $3.4 \mathrm{~mm}$ and the Expancel dimensions were in the range $20-40 \mu \mathrm{m}$. The reductions in the density and acoustic impedance are varying approximately linearly with the filler content. The minimum acoustic impedance reached is 0.88 MRayls, which is low enough for the design of a matching layer for an air coupled piezoelectric composite transducer [3]. The increasing levels of attenuation in the microsphere filled materials will affect the performance of such a matching layer. Therefore, it is necessary to balance the reduction in acoustic impedance with the increasing attenuation. Yano et al [3] have demonstrated 
improved air coupled transducers employing matching layer materials with acoustic attenuation in the range $3000-10000 \mathrm{~dB} / \mathrm{m}$, significantly higher levels of acoustic attenuation than observed in the current context.

As has already been stated, visual observations of the microsphere filled materials indicated that phase separation occurred at low microsphere loadings. The acoustic characterisation method employed, essentially measures the properties of the bulk of the material, with any variations in the formulation averaged out in the measurement. In order to investigate the properties of the materials in more detail, DMTA of these samples was carried out. The traces are shown in Figure 9.

\begin{tabular}{|c|c|c|c|c|c|}
\hline Sample & $\begin{array}{c}1 \text { wt \% } \\
(16 \% \text { Vol) }\end{array}$ & $\begin{array}{c}2 w t \% \\
\text { (27\% Vol) }\end{array}$ & $\begin{array}{c}3 \mathrm{wt} \% \\
\text { (36.5\% Vol) }\end{array}$ & $\begin{array}{c}4 \mathrm{wt} \% \\
(43.4 \% \mathrm{Vol})\end{array}$ & $\begin{array}{c}5 \text { wt \% } \\
(49 \% \text { Vol) }\end{array}$ \\
\hline $\mathrm{c}_{\mathrm{l}}\left(\mathrm{m} \cdot \mathrm{s}^{-1}\right)$ & 1691.3 & 1718.5 & 1439.8 & 1481.8 & 1486.7 \\
\hline $\mathrm{c}_{\mathrm{s}}\left(\mathrm{m} \cdot \mathrm{s}^{-1}\right)$ & 904.7 & 824.4 & 771.5 & 762.2 & 794.4 \\
\hline Density $\left(\mathbf{k g} \cdot \mathrm{m}^{-3}\right)$ & 1032 & 820 & 678 & 650 & 595 \\
\hline Poisson Ratio & 0.300 & 0.351 & 0.299 & 0.320 & 0.300 \\
\hline Z (MRayls) & 1.745 & 1.41 & 0.976 & 0.963 & 0.885 \\
\hline E (GPa) & 2.20 & 1.51 & 1.05 & 0.997 & 0.976 \\
\hline B (GPa) & 1.83 & 1.68 & 0.867 & 0.923 & 0.815 \\
\hline G (GPa) & 0.844 & 0.557 & 0.404 & 0.378 & 0.376 \\
\hline$\alpha_{1}\left(\mathrm{~dB} \cdot \mathrm{m}^{-1}\right)$ & 695 & 695 & 868 & 1303 & 1303 \\
\hline$\alpha_{s}\left(d B \cdot m^{-1}\right)$ & 1477 & 1780 & 2172 & 2172 & 2302 \\
\hline
\end{tabular}

Table 2 Mechanical properties of the CHDG-low density filler mixture determined by through transmission methodology at $500 \mathrm{kHz}$ 
From Figure 9 two transitions are observed in these materials; the higher temperature peak, observed at approximately $100{ }^{\circ} \mathrm{C}$, is associated with the Tg of the PVDF shell of the microsphere.
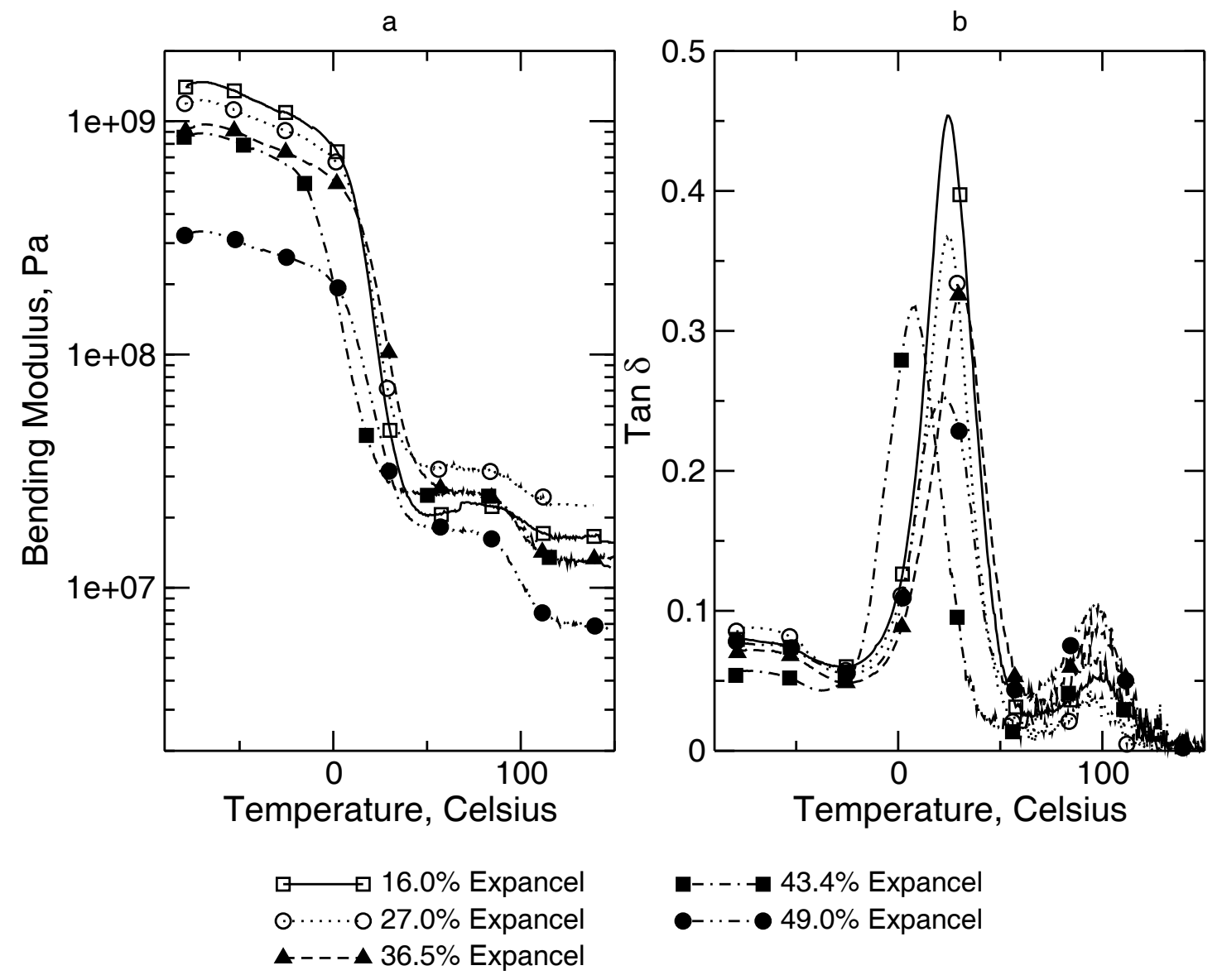

Figure 9 DMTA traces of CHDG with low-density filler. (a) bending modulus, (b) tan $\delta$

The lower temperature peak is that of the CHDG resin. The single peak associated with the CHDG resin indicates that the microspheres were evenly dispersed though the polymer system. As has already been stated, this was achieved by pre-polymerising the resin to increase the viscosity and so prevent separation of the two phases. A dual peak in this region of the DMTA would indicate phase separation, with one peak being related to the resin rich part of the material and the other a microsphere rich part of the sample. Therefore, the homogeneity of the materials is inferred from the DMTA trace. As the percentage of the filler is increased, both the low and high temperature moduli of the 
materials decrease. In general the $\mathrm{Tg}$ of the different systems are similar, but $\mathrm{Tg}$ is lowered significantly once the addition of the microspheres is increased above $43 \%$ volume fraction.

\section{HIGH DENSITY FILLER}

Attaining polymer-particle systems with a range of acoustic impedance is of interest in a number of applications in transducer design and manufacture, examples of which have been previously described in the preceding sections of this paper. Typically, such materials comprise tungsten particles in a polymer matrix with high values of specific acoustic impedance being attained [12]. Particle loaded polymer systems incorporating other metallic or metallic oxide particles have been reported in the literature [38, 39]. In the current context the use of tungsten to modify the impedance of the base resin was not possible since it inhibited the photocure of the polymer. In an attempt to achieve polymer particle composites exhibiting increased acoustic impedance, a high-density inorganic salt, $\mathrm{BaSO}_{4}$ having a density of $4400 \mathrm{~kg} \cdot \mathrm{m}^{-3}$, was incorporated into the BisA homopolymer. $\mathrm{BaSO}_{4}$ was selected since it is readily dispersed in the monomer and has a low UV absorbance and hence will not impede the cure of the resin. This system allows for materials with varying volume fractions of filler to be realised. Samples with $\mathrm{BaSO}_{4}$ volume fraction in the range $13-39 \%$ were synthesised. Table 3 details the elastic properties of the materials determined by the through transmission method at $500 \mathrm{kHz}$. Considering the longitudinal wave velocity data in Table 3, at low volume fractions of $\mathrm{BaSO}_{4}$ the velocity initially decreases from that of the base resin. This is a consequence of the particle inclusions in the polymer causing the scattered propagating wave front to negotiate a more tortuous path than in the base resin. As the filler content is increased particle contiguity means that the energy will navigate a more straight forward path through the material and the longitudinal velocity will increase. This is a common phenomenon in such particle loaded systems [40]. The dominance of the density in determining the acoustic impedance leads to an increase of acoustic impedance with

increasing $\mathrm{BaSO}_{4}$ concentration, despite the reduction in the longitudinal velocity. The maximum value of the acoustic impedance obtained was 6.25 MRayls and, as can be seen 
from Figure 10, the variation in acoustic impedance as function of filler composition is approximately linear.

\begin{tabular}{|c|c|c|c|c|c|}
\hline $\begin{array}{l}\text { Filler volume } \\
\text { fraction (\%) }\end{array}$ & 13 & 19 & 25 & 31 & 39 \\
\hline$c_{1}\left(m \cdot s^{-1}\right)$ & 2368.4 & 2257.4 & 2416.9 & 2472.8 & 2519.2 \\
\hline $\mathrm{c}_{\mathrm{s}}\left(\mathrm{m} \cdot \mathrm{s}^{-1}\right)$ & 1153.2 & 1155.5 & 1195.5 & 1208.1 & 1281.0 \\
\hline Density (kg.m³) & 1624.8 & 1828.9 & 1990.6 & 2213.7 & 2486.6 \\
\hline Poisson Ratio & 0.34 & 0.32 & 0.34 & 0.34 & 0.33 \\
\hline Z (MRayls) & 3.84 & 4.13 & 4.81 & 5.47 & 6.26 \\
\hline E (GPa) & 5.81 & 6.46 & 7.61 & 8.68 & 10.8 \\
\hline B (GPa) & 6.23 & 6.06 & 7.83 & 9.23 & 10.3 \\
\hline G (GPa) & 2.16 & 2.44 & 2.85 & 3.23 & 4.08 \\
\hline$\alpha_{1}\left(d B . m^{-1}\right)$ & 63 & 61 & 78 & 117 & 200 \\
\hline$\alpha_{s}\left(d B \cdot m^{-1}\right)$ & 243 & 243 & 208 & 191 & 243 \\
\hline
\end{tabular}

Table 3 Mechanical and acoustic properties of the $\mathrm{BaSO}_{4}$ loaded BisA samples determined by through transmission method at $500 \mathrm{kHz}$ 


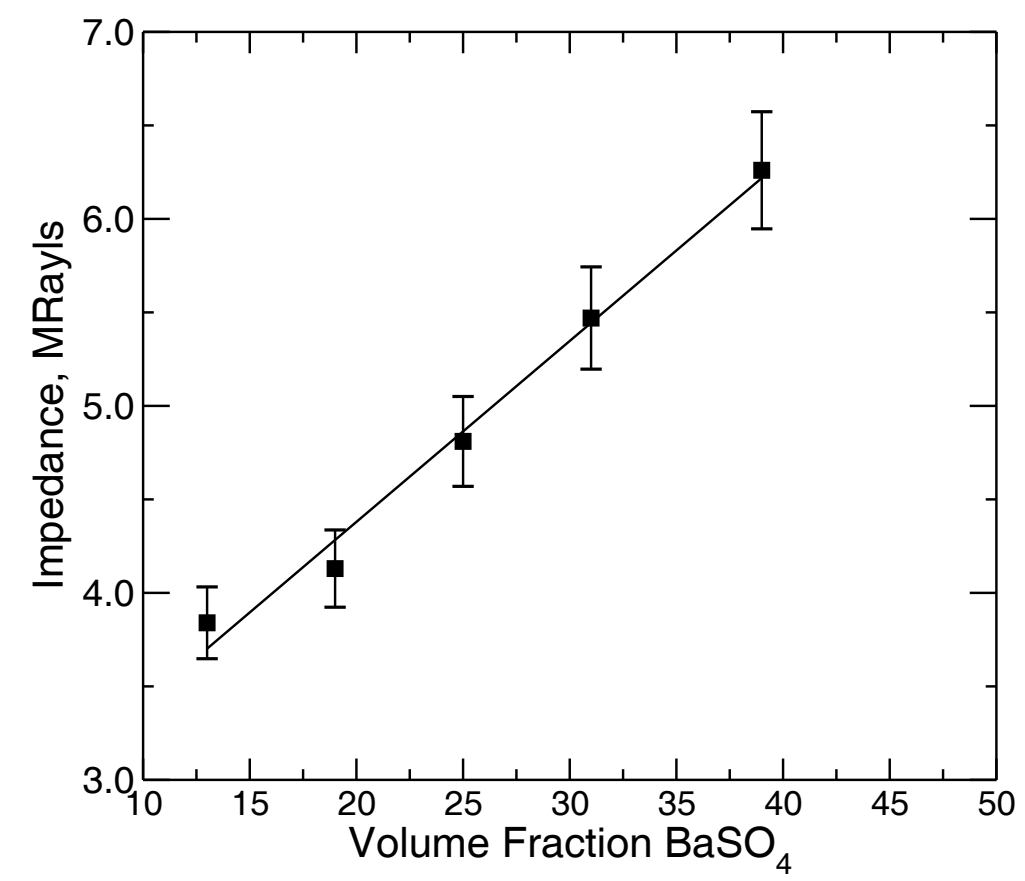

Figure 10 Variation in specific acoustic impedance as a function of filler volume fraction

- Acoustic Impedance and - linear fit determined by least squares method.

Error bars determined by propagation of uncertainty

Further investigation of the $\mathrm{BaSO}_{4}$ loaded polymer was undertaken using DMTA and DSC. Figure 11 details the DMTA data, where the addition of the $\mathrm{BaSO}_{4}$ lowers the low temperature modulus but increases the modulus above the glass transition temperature of the material. It appears that the $\mathrm{BaSO}_{4}$ is able to disrupt the formation of the highly crosslinked epoxy matrix leading to a lowering of the low temperature modulus, but the interactions between the resin and the filler enhance the modulus in the rubbery region. The DSC analysis, presented in Figure 12, indicates the presence of a postcure peak in the 13,19 and $25 \%$ volume fraction samples just above $\mathrm{Tg}$, indicative of uncured epoxy groups remaining in the matrix and consistent with the hypothesis that the lower modulus is a consequence of the $\mathrm{BaSO}_{4}$ inhibiting the cure process. The $\mathrm{BaSO}_{4}$ particles are able 
to interact with the polymer and hence effectively enhance the crosslink density in the rubbery phase with the net result of increased high temperature modulus being observed.

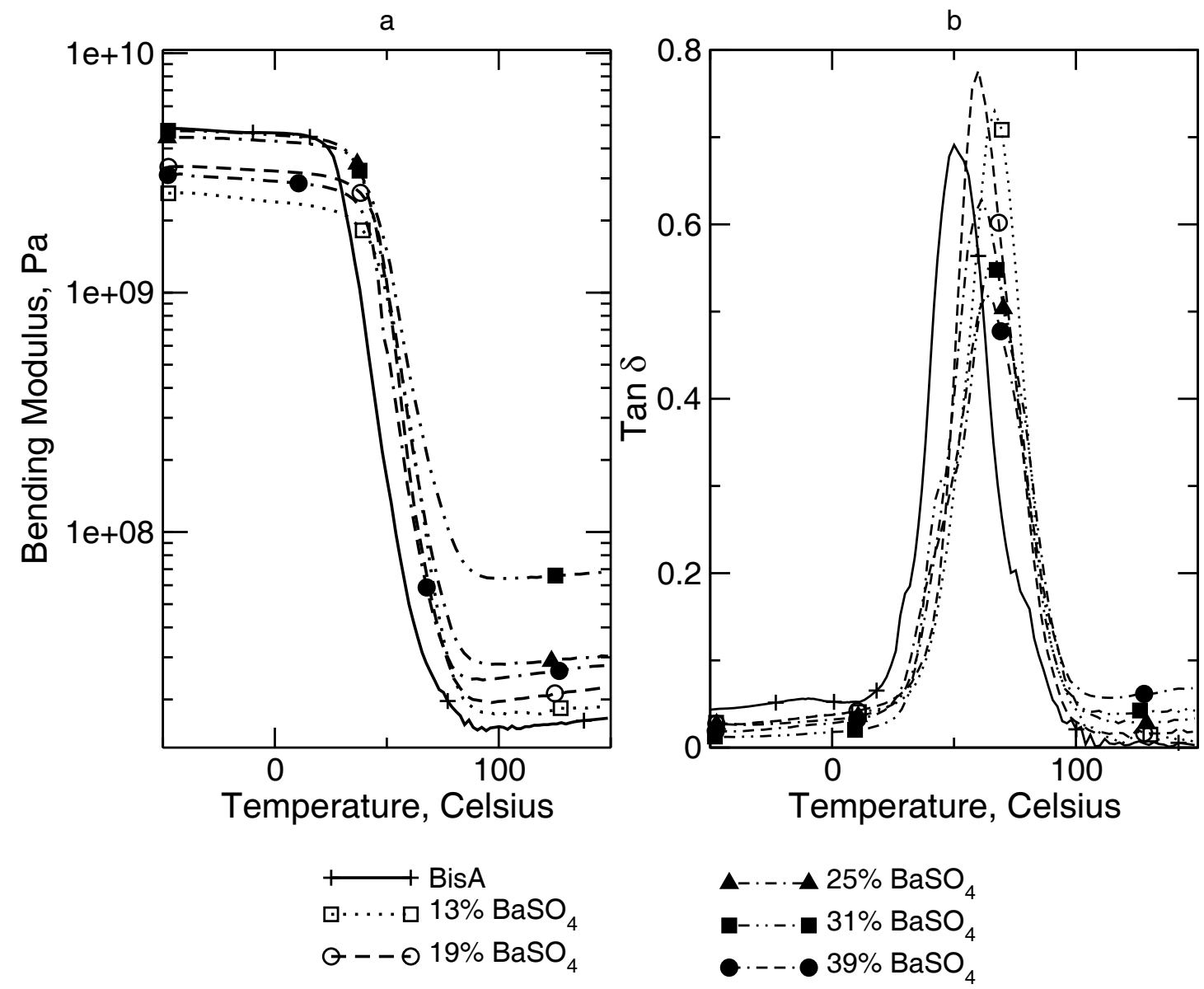

Figure 11 DMTA traces of BisA with high-density filler; (a) bending modulus, (b) tan $\delta$ 


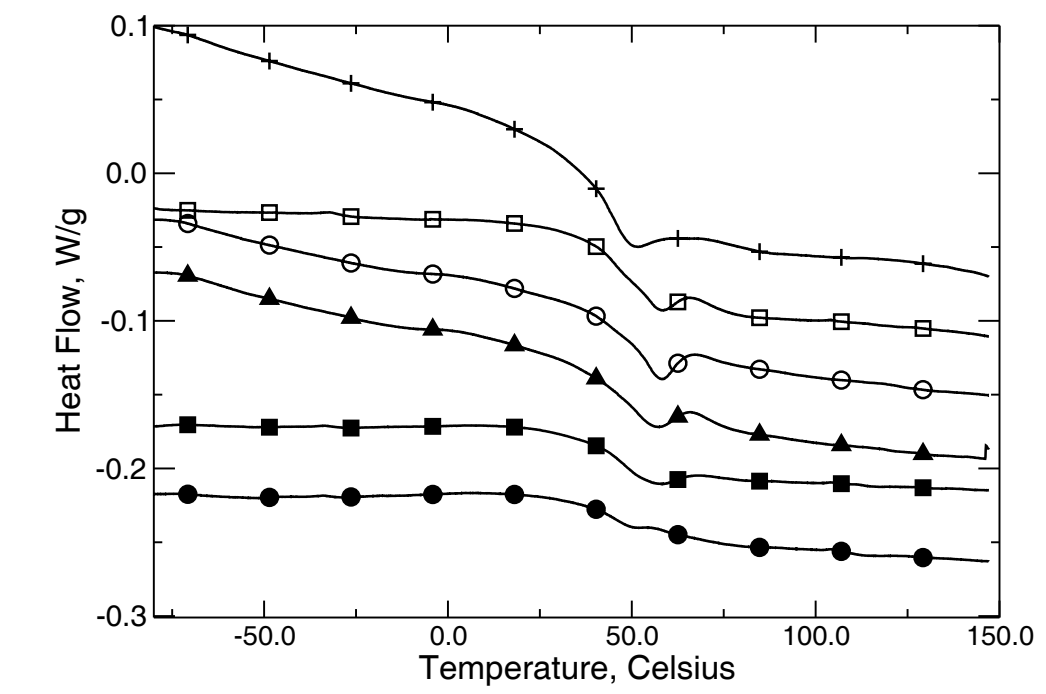

Figure 12 DSC traces of $\mathrm{BaSO}_{4}$ loaded BisA

+ BisA polymer , filler volume fraction: $\square 13 \%$, ○ 19\%, $\boldsymbol{\Delta} 25, \boldsymbol{\Delta} 31 \%, 039 \%$.

\section{CONCLUDING REMARKS}

This paper has presented the ultrasonic characterisation of polymer materials by a through transmission methodology. The method described, employs time of flight to initially determine the longitudinal and shear acoustic properties of isotropic samples of polymer. A method to determine the attenuation of each wave type is then described using a curve fitting method, based on the transmission coefficients of a liquid coupled isotropic polymer layer, to each of the propagating waves. The polymer samples investigated are cured via a novel UV initiated method and a variety of samples have been prepared including homopolymers, blends of different monomers and filled systems comprising either $\mathrm{BaSO}_{4}$ particles or polymer microspheres. Such materials are of interest to the transducer design community since it has been demonstrated that by combining blends of two photocurable epoxy monomers and the incorporation of hollow and dense fillers it is possible to adjust the acoustic impedance of the material over a significant range of values. For each of the systems studied, DMTA and DSC have been employed to further analyse the mechanical behaviour. In the case of the particle and microsphere filled materials DMTA and DSC have been used to elucidate the interaction of the filler 
material with the host polymer resin. The flexibility of one of the monomers, CHDG, gives rise to a significant increase in the acoustic attenuation which needs to be recognised when incorporating these materials into a probe design. Moreover, the scattering effects of the highly loaded hollow spheres must also be recognised. Finally, the $\mathrm{BaSO}_{4}$ loaded materials where found to exhibit a reduction in the crosslink density as a result of the particles inhibiting the course of the reaction. Post-cure peaks were observed in the DSC analysis of some of the samples, this phenomenon should be recognised when constructing probes and the ancillary layers using such particle filled materials.

The materials described exhibit a wide range of specific acoustic impedance, ranging from 0.88 MRayls to 6.25 MRayls and as such are suitable for a wide variety of ultrasonic matching layer applications. Moreover, since these materials can all be created by UV-initiated polymerisation, the samples presented have been formed in thin film format with thicker layers created by multiple progressive layer deposition. In principle, it is feasible to employ spin coating methods to create layers with variable thickness and acoustic impedance such that an impedance gradient from the transducer to the acoustic load can be achieved. This is the subject of further study by the authors.

\section{Acknowledgements}

The financial support of the UK Engineering and Physical Sciences Research Council under GR/S31235/01 to undertake this study, including the provision of the studentship for Alexandre Trogé, is acknowledged. The authors also wish to acknowledge the diligence of Mr Grant Smillie in undertaking the acoustic characterisation measurements. 


\section{References}

1. C.S. DeSilets, J.D. Fraser, and G.S. Kino, "The Design of Efficient Broad- Band Piezoelectric Transducers", IEEE Transactions on Sonics and Ultrasonics, Vol. SU-25, No. 3, pp. 115-125, (1978)

2. J.M. Cannata, T.A. Ritter, W.H. Chen, R.H. Silverman and K.K. Shung, 'Design of Efficient, Broadband Single Element (20-80MHz) Ultrasonic Transducers for Medical Imaging Applications' IEEE Transactions on Ultrasonics Ferroelectrics and Frequency Control, Vol. 50, No. 11, pp. 1548-1557, (2003)

3. T. Yano, M. Tone, and A. Fukumoto, 'Range finding and surface characterisation using high frequency air transducers' IEEE Transactions on Ultrasonics, Ferroelectrics and Frequency Control, Vol. 34 No2, pp. 232-236, (1987)

4. S.P. Kelly, G. Hayward, and T.E. Gómez Alvarez-Arenas, 'Characterisation and Assessment of an integrating matching layer for Air-coupled ultrasonic applications' IEEE Transactions on Ultrasonics, Ferroelectrics and Frequency Control, Vol. 51, No 10, pp. 1314-1323, (2004)

5. O. Krauss, R. Gerlach, and J. Fricke, 'Experimental and theoretical investigations of $\mathrm{SiO}_{2}$-aerogel matched piezoelectric transducers', Ultrasonics, Vol. 32 No. 3, pp. 217-222, (1994)

6. T.E. Gómez, F. Montero, E. Rodriguez, A., Roig, and E. Molins, 'Fabrication and characterisation of silica aerogel films for air-coupled piezoelectric transducers in the megahertz range' Proceedings 2002 IEEE Ultrasonics Symposium, pp. 1107-1110, (2002) 
7. P.C. Pedersen, O. Tretiak and P. He, 'Impedance-matching properties of an inhomogeneous matching layer with continuously changing acoustic impedance' Journal of the Acoustical Society of America, Vol. 72, pp. 327-336, (1982)

8. S.N. Ramadas, G. Hayward, R.L. O'Leary, T. McCunnie, A.J. Mulholland, A. Trogé, R.A. Pethrick, D. Robertson, and V. Murray, 'A Three-Port Acoustic Lattice Model for Piezoelectric Transducers Containing Opposing Zones of Polarization' Proceedings of the 2006 IEEE International Ultrasonics Symposium, pp. 1899-1902, (2006)

9. A.J. Mulholland, S.N. Ramadas, R.L. O'Leary, , A.C.S. Parr, , A. Trogé, R.A. Pethrick and G. Hayward, 'Enhancing the performance of piezoelectric ultrasound transducers by the use of multiple matching layers' IMA Journal of Applied Mathematics, Vol. 73, pp. 936-949, (2008)

10. H. Tohmyoh, 'Polymer acoustic matching layer for broadband ultrasonic applications', Journal of the Acoustical Society of America, Vol. 120, pp. 31-34 (2006)

11. R.L. O'Leary, A.C.S. Parr, G. Hayward and G. Smillie, 'CUE Materials Database' [Online] http://www.cue.ac.uk/cue_materials_database_ver1.2_aug_200 5.pdf - (date last viewed May 17th 2010)

12. M.G. Grewe, T.R. Gururaja, T.R. Shrout and R.E. Newnham, 'Acoustic Properties of Particle/Polymer Composites for Ultrasonic Transducer Backing Applications' IEEE Transactions on Ultrasonics, Ferroelectrics and Frequency Control, Vol. 37, No. 6, pp. 506-514, (1990)

13. H. Wang, T.A. Ritter, W. Cao and K.K. Shung, 'High Frequency Properties of Passive Materials for Ultrasonic Transducers' IEEE Transactions on Ultrasonics, Ferroelectrics and Frequency Control, Vol. 48, No. 1, pp. 78-84, (2001) 
14. K. Saito, K. Yamaguchi and M. Kawabuchi, 'Piezoelectric ultrasonic probe using and epoxy resin and iron carbonyl acoustic matching layer' United States Patent number 4,616,152, (October 7, 1986)

15. A. Trogé, 'A new range of ultrasonic transducers' Ph.D. Thesis, University of Strathclyde, Glasgow

16. A.R. Mackintosh, A.J.C. Kuehne, R.A. Pethrick, B. Guilhabert, E. Gu, C.L. Lee, M.D. Dawson, G. Heliotis and D.D.C. Bradley 'Novel polymer systems for deep UV microlens arrays' Journal of Physics D: Applied Physics, Vol. 41, No. 9, Article 094007, (2008)

17. S. Dixon, D. Jacques, S. D. Palmer and G. Rowlands, 'The measurement of shear and compression waves in curing epoxy adhesives using ultrasonic reflection and transmission techniques simultaneously' Measurement Science and Technology Vol. 15, pp. 939-947, (2004)

18. F. El-Tantawy and Y.K. Sung, 'A novel ultrasonic transducer backing from porous epoxy resin-titanium-silane coupling agent and plasticizer composites' Materials Letters Vol. 58, pp. 154-158, (2003)

19. B. Faiz, G. Maze, D. Decoultot, A. Moudden, E. H. Assif and M. Ezzaidi, 'Ultrasonic Characterization of the Quality of an Epoxy Resin Polymerization' IEEE Transactions on Ultrasonics, Ferroelectrics and Frequency Control, Vol. 46, pp. 188-196, (1999)

20. S. Dixon and B. Lanyon, 'Phase change measurement of ultrasonic shear waves on reflection from a curing epoxy system' Journal of Physics D: Applied Physics, Vol. 38 , pp. 4115-4125, (2005)

21. L. G. Bunton, J. H. Daly, I. D. Maxwell and R. A. Pethrick, 'Investigation of cure in epoxy-resins - ultrasonic and thermally stimulated current measurements' Journal of Applied Polymer Science, Vol. 27, pp. 4283-4294, (1982) 
22. M. Frigione, A. Maffezzoli, D. Acierno, V. Luprano and G. Montagna, 'Nondestructive and in-situ monitoring of mechanical property build up in epoxy adhesives for civil applications by propagation of ultrasonic waves' Polymer Engineering and Science, Vol. 40, pp. 656-664, (2000)

23. K. Liang, H. Kunkel, C. Oakley and W. Huebner, 'Acoustic characterisation of ultrasonic transducer materials: I. Blends of rigid and flexible epoxy resins used in piezocomposites' Ultrasonics, Vol. 36, No. 10, pp. 979-986, (1998)

24. R.E. Montgomery, F.J. Weber, D.F. White and C.M. Thompson, 'On the development of acoustically transparent structural plastics' Journal of the Acoustical Society of America, Vol. 71, No. 3, pp. 735-741, (1982)

25. J. Krautkrämer, and H. Krautkrämer, Ultrasonic Testing of Materials. Second Edition; Springer-Verlag: Berlin; New York, pp. 528-550, (1977)

26. V.A. Shutilov, Fundamental Physics of Ultrasound. Gordon and Breach Science Publishers, New York, pp. 211-216, (1988),

27. R.J. Freemantle and R.E. Challis, 'Combined compression and shear wave ultrasonic measurements on curing adhesive', Measurement Science and Technology, Vol. 9, pp. 1291-1302, (1998)

28. R.L. O'Leary, 'An Investigation into the Passive Polymer Materials Utilised in the Construction of Piezoelectric Composite Transducers' PhD. Thesis, University of Strathclyde, Glasgow, Scotland, (2003).

29. A.R., Selfridge 'Approximate Material Properties in Isotropic Materials', IEEE Transactions on Sonics and Ultrasonics, Vol. SU-32, No. 3, (1985)

30. L.M. Brekhovskikh and O.A. Godin Acoustics of Layered Media I, Springer Verlag, Berlin, Second Edition, pp. 94-98, (1998) 
31. B.A. Auld, Acoustic Fields and Waves in Solids, Vol. 1, Krieger Publishing Company, Florida, pp. 86-99, (1990)

32. H. Kolsky, Stress Waves in Solids, New York: Dover Publications, pp. 116-122, (1963)

33. J.R. Taylor, An introduction to error analysis : the study of uncertainties in physical measurements University Science Books, Sausalito, CA, pp. 45-91, (1997)

34. A.C.S. Parr, R.L. O'Leary and G. Hayward, 'Improving the thermal stability of piezoelectric composite transducers', IEEE Transactions on Ultrasonics, Ferroelectrics and Frequency Control, Vol. 52, No. 4, pp. 550-563, (2005)

35. J. Heijboer, 'The Movement of the Cyclohexyl Group in Glassy Polymers' Kolloid Zeitschrift, Vol. 171, pp. 7-15 (1960)

36. P.K. Datta and R.A. Pethrick, 'Ultrasonic Studies of Glass-Filled Polymer Solids' Journal of Physics D - Applied Physics, Vol. 13, pp. 153-161, (1980)

37. R.E. Challis, A.K. Holmes, J.S. Tebbutt and R.P. Cocker, 'Scattering of ultrasonic compression waves by particulate filler in a cured epoxy continuum' Journal of the Acoustical Society of America, Vol. 103, pp. 1413-1420, (1998)

38. Y. Hosono, Y. Yamashita and K. Itsumi, 'Effects of metal particle dopant on acoustic attenuation properties of silicone rubber lens for medical echo array probe' Japanese Journal of Applied Physics, Vol. 44, No. 6B, pp. 4558-4560, (2005)

39. Y. Hosono, Y. Yamashita and K. Itsumi, 'Effects of fine metal oxide particle dopant on acoustic properties of silicone rubber lens for medical array probe' IEEE Transactions on Ultrasonics, Ferroelectrics and Frequency Control, Vol. 54, No 8, pp. 796-799, (2007) 
40. T.E. Gómez Alvarez-Arenas, A.J. Mulholland, G. Hayward and J. Gomatam, 'Wave propagation in 0-3/3-3 connectivity composites with complex microstructure', Ultrasonics, Vol. 38, No. 9, pp. 897-907, (2000) 
Table 1 Mechanical and acoustic properties of the BisA-CHDG mixture polymers determined by through transmission where $c_{l}$ is the longitudinal velocity, $c_{s}$ the shear velocity, $Z$ the acoustic impedance, E the Young modulus, $K$ the bulk modulus, $G$ the shear modulus, $\alpha_{l}$ the longitudinal attenuation and $\alpha_{s}$ the shear attenuation

\begin{tabular}{|c|c|c|c|c|c|c|c|c|c|c|c|}
\hline $\begin{array}{c}\text { Formulation } \\
\text { Volume Fraction of } \\
\text { BisA in CHDG }\end{array}$ & $\mathbf{0}$ & 9.8 & 19.7 & 29.6 & 40.0 & 50.0 & 59.6 & 69.6 & 79.7 & 89.8 & 100 \\
\hline$c_{l}\left(m \cdot s^{-1}\right)$ & 1784.8 & 1956.3 & 2003.2 & 2091.0 & 2232.6 & 2246.9 & 2356.8 & 2392.7 & 2441.4 & 2531.0 & 2568.3 \\
\hline$c_{s}\left(m \cdot s^{-1}\right)$ & 766.3 & 800.4 & 799.5 & 870.7 & 910.5 & 924.0 & 943.8 & 971.9 & 1004.7 & 1048.5 & 1092.7 \\
\hline Density $\left(\mathrm{kg} \cdot \mathrm{m}^{-3}\right)$ & 1157.3 & 1156.0 & 1158.8 & 1152.9 & 1162.0 & 1172.4 & 1178.0 & 1181.0 & 1170.8 & 1180.9 & 1177.9 \\
\hline Poisson's Ratio & 0.387 & 0.400 & 0.405 & 0.395 & 0.400 & 0.398 & 0.405 & 0.401 & 0.398 & 0.396 & 0.390 \\
\hline Z (MRayls) & 2.066 & 2.261 & 2.321 & 2.411 & 2.594 & 2.634 & 2.776 & 2.826 & 2.858 & 2.989 & 3.025 \\
\hline E (GPa) & 1.885 & 2.073 & 2.082 & 2.439 & 2.698 & 2.799 & 2.948 & 3.126 & 3.305 & 3.626 & 3.908 \\
\hline K (GPa) & 2.780 & 3.437 & 3.662 & 3.875 & 4.508 & 4.584 & 5.144 & 5.274 & 5.403 & 5.834 & 5.894 \\
\hline G (GPa) & 0.679 & 0.741 & 0.741 & 0.8740 & 0.963 & 1.001 & 1.049 & 1.116 & 1.182 & 1.298 & 1.406 \\
\hline$\alpha_{1}\left(\mathbf{d B} \cdot \mathrm{m}^{-1}\right)$ & 573 & 426 & 252 & 174 & 156 & 130 & 52 & 43 & 26 & 35 & 35 \\
\hline$\alpha_{\mathrm{s}}\left(\mathrm{dB} \cdot \mathrm{m}^{-1}\right)$ & 6228 & 5993 & 4334 & 3839 & 2562 & 2493 & 1729 & 1416 & 973 & 643 & 356 \\
\hline $\operatorname{Tg}\left({ }^{\circ} \mathbf{C}\right)$ & $23 \pm 1$ & $30 \pm 1$ & $35 \pm 1$ & $44 \pm 1$ & $48 \pm 1$ & $52 \pm 1$ & $57 \pm 1$ & $61 \pm 1$ & $65 \pm 1$ & $66 \pm 1$ & $62 \pm 1$ \\
\hline
\end{tabular}


Table 2 Mechanical properties of the CHDG-low density filler mixture determined by through transmission methodology at $500 \mathrm{kHz}$

\begin{tabular}{|c|c|c|c|c|c|}
\hline Sample & $\begin{array}{c}1 \text { wt \% } \\
(16 \% \text { Vol) }\end{array}$ & $\begin{array}{c}2 w t \% \\
(27 \% \text { Vol) }\end{array}$ & $\begin{array}{c}3 \text { wt \% } \\
(36.5 \% \text { Vol) }\end{array}$ & $\begin{array}{c}4 \text { wt \% } \\
(43.4 \% \mathrm{Vol})\end{array}$ & $\begin{array}{c}5 \text { wt \% } \\
(49 \% \text { Vol) }\end{array}$ \\
\hline $\mathrm{C}_{\mathrm{l}}\left(\mathrm{m} \cdot \mathrm{s}^{-1}\right)$ & 1691.3 & 1718.5 & 1439.8 & 1481.8 & 1486.7 \\
\hline $\mathrm{c}_{\mathrm{s}}\left(\mathrm{m} \cdot \mathrm{s}^{-1}\right)$ & 904.7 & 824.4 & 771.5 & 762.2 & 794.4 \\
\hline Density $\left(\mathbf{k g} \cdot \mathrm{m}^{-3}\right)$ & 1032 & 820 & 678 & 650 & 595 \\
\hline Poisson Ratio & 0.300 & 0.351 & 0.299 & 0.320 & 0.300 \\
\hline Z (MRayls) & 1.745 & 1.41 & 0.976 & 0.963 & 0.885 \\
\hline E (GPa) & 2.20 & 1.51 & 1.05 & 0.997 & 0.976 \\
\hline B (GPa) & 1.83 & 1.68 & 0.867 & 0.923 & 0.815 \\
\hline G (GPa) & 0.844 & 0.557 & 0.404 & 0.378 & 0.376 \\
\hline$\alpha_{1}\left(\mathrm{~dB} \cdot \mathrm{m}^{-1}\right)$ & 695 & 695 & 868 & 1303 & 1303 \\
\hline$\alpha_{s}\left(d B \cdot m^{-1}\right)$ & 1477 & 1780 & 2172 & 2172 & 2302 \\
\hline
\end{tabular}


Table 3 Mechanical and acoustic properties of the $\mathrm{BaSO}_{4}$ loaded BisA samples determined by through transmission method at $500 \mathrm{kHz}$

\begin{tabular}{|c|c|c|c|c|c|}
\hline $\begin{array}{l}\text { Filler volume } \\
\text { fraction (\%) }\end{array}$ & 13 & 19 & 25 & 31 & 39 \\
\hline$c_{1}\left(m \cdot s^{-1}\right)$ & 2368.4 & 2257.4 & 2416.9 & 2472.8 & 2519.2 \\
\hline $\mathrm{c}_{\mathrm{s}}\left(\mathrm{m} \cdot \mathrm{s}^{-1}\right)$ & 1153.2 & 1155.5 & 1195.5 & 1208.1 & 1281.0 \\
\hline Density (kg.m ${ }^{-3}$ ) & 1624.8 & 1828.9 & 1990.6 & 2213.7 & 2486.6 \\
\hline Poisson Ratio & 0.34 & 0.32 & 0.34 & 0.34 & 0.33 \\
\hline Z (MRayls) & 3.84 & 4.13 & 4.81 & 5.47 & 6.26 \\
\hline E (GPa) & 5.81 & 6.46 & 7.61 & 8.68 & 10.8 \\
\hline B (GPa) & 6.23 & 6.06 & 7.83 & 9.23 & 10.3 \\
\hline G (GPa) & 2.16 & 2.44 & 2.85 & 3.23 & 4.08 \\
\hline$\alpha_{1}\left(\mathrm{~dB} \cdot \mathrm{m}^{-1}\right)$ & 63 & 61 & 78 & 117 & 200 \\
\hline$\alpha_{s}\left(d B \cdot m^{-1}\right)$ & 243 & 243 & 208 & 191 & 243 \\
\hline
\end{tabular}


Figure 1 Chemical structures of the epoxy resins and the photo-initiator

Figure 2 Photograph of the experimental apparatus

Figure 3 Curve Fitting for the determination of attenuation in CHDG, upper plot - Longitudinal data and lower plot - Shear data

Figure 4 Comparison of experimentally determined moduli as the composition is varied,

- Bulk modulus, $\square$ Young's modulus, $\bullet$ Shear modulus and - data derived from the mixing rule.

Error bars determined by propagation of uncertainty

Figure 5 Variation in acoustic impedance of the polymer blend as the composition is varied

- Measured Acoustic Impedance and - Acoustic Impedance data derived from the mixing rule. Error bars determined by propagation of uncertainty

Figure 6 Variation in attenuation measured at $500 \mathrm{kHz}$ as a function of composition,

- Longitudinal Attenuation, Shear Attenuation and - linear fit determined by least squares method

Figure 7 DMTA traces of the BisA-CHDG mixtures measured at $1 \mathrm{~Hz}$;

(a) bending modulus and (b) Tan $\delta$.

Figure 8 DSC traces of the BisA-CHDG mixture measured at a temperature ramp of $10^{\circ} \mathrm{C} / \mathrm{min}$.

$+100 \%$ CHDG, $19.7 \%$ BisA, $\square$ 40\% BisA, \ 59.6\% BisA, O 79.7\% BisA, 口 100\% BisA.

Figure 9 DMTA traces of CHDG with low-density filler. (a) bending modulus, (b) tan .

Figure 10 Variation in specific acoustic impedance as a function of filler volume fraction

- Acoustic Impedance and - linear fit determined by least squares method.

Error bars determined by propagation of uncertainty

Figure 11 DMTA traces of BisA with high-density filler; (a) bending modulus, (b) tan $\delta$

Figure 12 DSC traces of $\mathrm{BaSO}_{4}$ loaded BisA

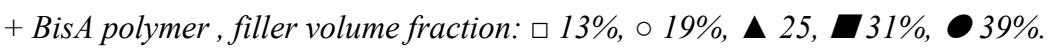

